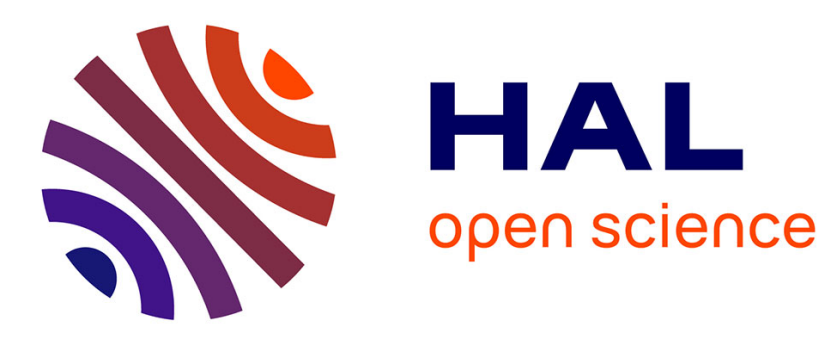

\title{
Les Spécificités de la Gouvernance des Entreprises Familiales: Cas NCA Rouiba
}

Hichem Sofiane Salaouatchi, Kamila Ait Yahia, Adja Hamida

\section{To cite this version:}

Hichem Sofiane Salaouatchi, Kamila Ait Yahia, Adja Hamida. Les Spécificités de la Gouvernance des Entreprises Familiales: Cas NCA Rouiba. Journal Of North African Economies, 2019, 15 (1), pp.391-403. 10.33858/0470-000-020-025 . hal-03214675

\section{HAL Id: hal-03214675 \\ https://hal.science/hal-03214675}

Submitted on 2 May 2021

HAL is a multi-disciplinary open access archive for the deposit and dissemination of scientific research documents, whether they are published or not. The documents may come from teaching and research institutions in France or abroad, or from public or private research centers.
L'archive ouverte pluridisciplinaire HAL, est destinée au dépôt et à la diffusion de documents scientifiques de niveau recherche, publiés ou non, émanant des établissements d'enseignement et de recherche français ou étrangers, des laboratoires publics ou privés. 


\title{
les specificites de la gouvernance des entreprises familiales cas : nca rouiba the specificities of the governance of family entreprises case: $n$ ca rouiba
}

\author{
Dr.salaouatchi Hichem Sofiane \\ Eocle des Hautes Etudes \\ Commerciales (HEC), \\ Laboratoire MPI-Algérie \\ hs.salaouatchi@hec.dz
}

\author{
Dr.AIT YAHIA KAMILA \\ Ecole Nationale Supérieure de \\ Management (ENSM)- Algérie \\ k.aityahia@ensm.dz
}

\author{
E.D ADJA Hamida \\ Doctorante à l'école Supérieure \\ de commerce-Algérie \\ hecchekirine@gmail.com
}

Received: $15 / 07 / 2018$

Published: 05/03/2019

\begin{abstract}
:
Corporate governance has received the most attention in recent years. While this new practice is extensively discussed, it is not always well understood, especially at family enterprises. Bearing these are the oldest and most widespread form of business organisation in the world and play a leading role in economic growth and employment of the workforce. As a result, in this study we focused on highlighting the main characteristics of the family enterprise compared to the managerial enterprise in terms of its governance and structures.

Key words: Corporate governance, family entreprise, family governance, family governance
\end{abstract} structures.

\section{Introduction :}

La gouvernance d'entreprise est devenue incontournable pour l'entreprise familiale, du fait de certaines spécificités qui sont dues principalement aux interactions famille/entreprise, l'intérêt de la gouvernance est double, car en plus des bienfaits de la mise en place d'un système de gouvernance reconnu pour toute entreprise, il y a également le souci de l'organisation et l'harmonie de la famille comme enjeu. Nous pensons que nous ne pouvons pas comprendre la gouvernance des entreprises familiales sans une réelle prise en compte de la dimension familiale, des mécanismes qui régissent la famille et son évolution.

Ainsi, au cours du développement du présent travail de recherche nous tenterons de répondre à la problématique suivante :

\section{Comment les entreprises familiales algériennes sont-elles gouvernées?}

Pour ce faire, nous allons présenter en premier lieu l'entreprise familiale, afin de mettre en avant certaines caractéristiques de cette dernière. Nous nous intéresserons par la suite à la gouvernance dans l'entreprise familiale et monter l'importance de la mise en place d'une gouvernance familiale qui est un nécessaire complément à la gouvernance d'entreprise afin d'assurer la pérennité de l'entreprise. En dernier nous allons analyser le mode de gouvernance de l'une des entreprises familiales algériennes leadeur dans le secteur agroalimentaire qui est la NCA Rouiba.

L'objectif de cette communication est de préparer l'entreprise familiale algérienne à la conduite de son changement vers la mise en œuvre des concepts de gouvernance et de ses exigences comportementales et structurelles.

2. Cadre conceptuel de l'entreprise familiale :

Les définitions présentées dans la littérature de gestion sont à la fois très nombreuses, et très marquées par l'hétérogénéité. Cela est dû au fait que l'entreprise familiale ne peut être appréhendée ni à travers des formes juridiques spécifiques ni à travers des tailles spécifiques.

Les trois principaux critères qui reviennent le plus souvent dans les différentes définitions, qui permettent d'identifier les entreprises familiales sont le contrôle du capital par la famille, la participation de la famille dans la gestion de l'entreprise et la transmission de l'entreprise à la génération suivante.

1 - Corresponding author: SALAOUATCHI Hichem Sofiane, e-mail:hs.salaouatchi@hec.dz 
Néanmoins, Ponza (2007) a proposé les critères rassemblent les entreprises familiales dans une définition commune :

- La détention d'un minimum de $15 \%$ du capital par au moins deux membres d'une même famille ou d'un groupe de familles ;

- L'influence déterminante sur la stratégie de l'entreprise exercée en faisant partie du management, en étant administrateurs, conseils ou actionnaires actifs, ou en contribuant à définir la culture d'entreprise ;

- L'importance accordée aux rapports entre les membres de la famille ;

- Le souhait ou la possibilité d'une transmission aux générations suivantes.

\section{La gouvernance familiale :}

La particularité du système de gouvernance des entreprises familiales a été soulignée par plusieurs chercheurs (Melin et Nordqvist, 2000 ; Carney (2005) ; Miller et Breton-Miller, 2006).

Pour Kenyon- Rouvinez et Ward (2004) : le gouvernement de l'entreprise familiale est un concept très particulier et différent de celui issu de la théorie de l'agence Il s'agit " un système de processus et de structures mis en place au plus haut niveau de l'entreprise, de la famille et de l'actionnariat, pour garantir les meilleures décisions concernant la direction, les responsabilité et le contrôle de l'entreprise ". Donc l'attention doit être portée à la famille, les échanges formels et informels entre ses membres et au pouvoir effectif qu'elle exerce sur l'entreprise moyennant le contrôle de la propriété, du conseil d'administration et de la gestion.

\section{Les conflits existants dans l'entreprise familiale:}

- Les conflits entre les besoins personnels et ceux de l'entreprise : Les intérêts de l'entreprise ne convergent pas toujours avec ceux des actionnaires qui ne travaillent pas en son sein. Ces derniers, connaissent mal ou peu la situation de l'entreprise, remettent en cause le bien-fondé de certaines décisions ou n'acceptent plus la politique de non-distribution des dividendes.

- Les conflits institutionnels : Dus au chevauchement institutionnel entre l'entreprise et la famille (Lansberg 1980).

- Les conflits de fonctions : Selon Barry (1980), des conflits peuvent surgis si le dirigeantpropriétaire assume trois fonctions différentes au sein de sa société : Propriétaire, administrateur et salarié.

5. L'entreprise familiale dans les débats théoriques:

Sur le plan de l'analyse théorique, le phénomène du « family business » étant un domaine de recherche assez récent, on assiste alors à une profusion de contributions, mais sans qu'il y ait de paradigme unifié (Wortman, 1994).

Ainsi, les auteurs qui empruntent la voie de la théorie de l'agence pour l'étude de l'entreprise familiale considèrent que dans celle-ci les conflits et les coûts d'agence seraient pratiquement nuls dans la mesure où les propriétaires étant souvent en même temps dirigeants, il y aurait un recoupement des intérêts quant à la logique de développement, de risque et de maximisation des profits. pour Jensen et Meckling (1976), les procédures de contrôle ne serviraient qu'à grever la rentabilité de l'entreprise familiale et ne seraient d'aucune utilité. De plus, la théorie de l'agence se focalise sur l'allocation de ressources financières (incitations, etc.) et ne prend que peu en compte les éléments non monétaires (motivations de la famille, projet familial). L'inapplicabilité stricte de cette dernière aux entreprises familiales - particulièrement les non cotées puisque le capital est relativement concentré a notamment été soulignée par Melin et Nordqvist en 2000.Salvato (2002) comme Davis, Schoorman et Donaldson (1997) suggèrent que la théorie la plus susceptible de s'appliquer à l'entreprise familiale est la théorie de l'intendance qui propose une modélisation du comportement humain dans laquelle l'individu se comporte comme un intendant qui retire une plus grande utilité de comportements organisationnels et coopératifs que de comportements individualistes et égoïstes. Ce cadre analytique semble mieux adapté à la compréhension des spécificités des entreprises familiales, dans la mesure où l'on peut supposer que le projet familial oriente l'action des membres de la famille impliqués dans l'entreprise. Tout particulièrement 
dans une PME familiale où la concentration du capital est forte. Au regard de cette théorie donc, il ne semble pas y avoir de bonnes raisons à la mise en place de structures formelles de gouvernement d'entreprise dans les PME familiales non cotées.

D'autres auteurs tels que Schulze, Lubatkin et ali. Ont pensé à élargir la théorie de l'agence en y incorporant des relations entre principaux et agents autres que simplement financières et monétaires. En particulier, ils pointent du doigt sur l'altruisme parental comme une «fonction d'utilité qui lie le bénéfice/richesse d'un individu à celui des autres » ce qui pousse le dirigeant propriétaire à être à l'écoute des membres de la famille, à obtenir l'adhésion à long terme des membres de la famille qui par leur multitude ont des opinions divergentes, à concilier les intérêts de la famille et la croissance de l'entreprise. Mais les entreprises familiales peuvent parfois être exposées à des risques d'agence malgré l'altruisme du dirigeant propriétaire : choix d'un mauvais successeur, rationalité limitée du décideur pouvant conduire à faire «mal » en voulant faire «bien ». Une famille n'est pas un ensemble de personnes homogènes de la même génération, ayant les mêmes intérêts, des conflits familiaux peuvent compromettre l'échange d'informations et la collaboration. L'altruisme entre membres de la famille et la recherche du bien-être général peut s'effacer pour laisser place à une recherche de profit personnel et une attitude opportuniste.

Ces voies de recherche récentes, qui incorporent la dimension affective et émotive dans la théorie de l'agence, nous permettent donc de revenir sur la question de l'intérêt possible de la mise en place d'institution de gouvernement d'entreprise dans les entreprises familiales non cotées : on peut en effet penser que divers comités, un conseil de famille, une charte familiale, seraient des institutions capables d' « objectiver » certaines décisions stratégiques devant être prises pour l'entreprise.

\section{Les étapes de la croissance d'une entreprise familiale:}

Plusieurs modèles ont été définis afin de décrire et d'analyser les différentes étapes que traversent les entreprises familiales au cours de leur existence. Le modèle basique de Ward (1991) à trois étapes résume le cycle de vie de l'entreprise familiale comme suit :

- Le(s) Fondateur(s) (Propriétaire(s) Détenant le Contrôle : Il s'agit de l'étape initiale dans l'existence d'une entreprise familiale. L'entreprise est alors entièrement dirigée par le(s) fondateur(s). Transition du poste de dirigeant, succession, planification sont les problèmes majeurs qui caractérisent cette étape.

- Le Partenariat raternel : Il s'agit de l'étape où la gestion et la propriété ont été transférées aux enfants du fondateur(s). Dans cette étapes, plusieurs problèmes de gouvernance peuvent être surgis tels que le maintien du travail d'équipe et de l'harmonie, prolongation de la propriété familiale et la succession

- La Confédération des Cousins (Consortium des Cousins ou Dynastie Familiale): Dans cette étape, la gouvernance d'entreprise devient plus complexe, étant donné que davantage de membres de la famille sont directement ou indirectement impliqués dans l'entreprise, y compris les enfants des frères et sœurs, les cousins et la belle-famille. De ce fait, on trouve une multitude de problèmes, à savoir :

- Attribution du capital de l'entreprise, niveaux des dividendes, dettes et bénéfices

- Liquidité des actions

- Résolution des conflits familiaux

- Participation et rôle de la famille

- Vision et mission de la famille

- Lien familial avec l'entreprise

7. Le rôle des membres de la famille dans la gouvernance de leur entreprise :

Dans une entreprise familiale typique, un individu peut avoir des rôles et des responsabilités multiples associés à des motivations différentes, ce qui augmente les défis auxquels les entreprises familiales doivent faire face, par opposition à leurs équivalents non familiales. 
- Les propriétaires : Parmi les rôles d'un propriétaire, on peut citer :

-propriétaire uniquement.

- propriétaire/ dirigeant.

- propriétaire/membre de la famille.

- propriétaire/membre de la famille/dirigeant.

- propriétaire/ administrateur.

- propriétaire/membre de la famille/administrateur

- Les dirigeants : Un des problèmes communs dans les entreprises familiales est la différence de traitement entre les dirigeants issus de la famille et les dirigeants extérieurs. Dans de nombreuses entreprises familiales, une partie où la totalité des postes de direction sont strictement réservés aux membres de la famille. Cela peut avoir des répercussions négatives sur la motivation et les résultats des cadres non issues de la famille. Définir une politique d'emploi claire et juste (à la fois pour les employés issus de la famille et pour ceux qui ne sont pas issus de la famille) aide les entreprises familiales à maintenir la motivation de leurs meilleurs employés et leur intérêt dans la croissance de l'entreprise.

- Les administrateurs : La plupart des entreprises familiales réservent ce droit aux membres de la famille et dans quelques cas à certains dirigeants non issus de la famille en lesquels ils ont une grande confiance. En effet la plupart des décisions sont prises par les administrateurs issus de la famille. Dans le cadre de l'exemple de la distribution de dividendes, les administrateurs familiaux qui travaillent dans l'entreprise encourageront le réinvestissent des bénéfices pour augmenter son potentiel de croissance. Contrairement, aux administrateurs familiaux qui ne travaillent pas dans l'entreprise et qui, préfèrent la distribution des bénéfices sous forme de dividendes aux actionnaires familiaux. Ces points de vue contradictoires peuvent aboutir à des conflits au sein du conseil d'administration.

- Les membres de la famille : Les membres de la famille peuvent avoir des responsabilités, des droits et des attentes différentes de la part de leur entreprise. Le degré d'accès aux informations reste le problème majeur pour ces derniers, en effet, les membres qui travaillent dans l'entreprise ont généralement accès à ce genre d'informations au moment opportun contrairement à ceux qui ne font pas partie de l'entreprise. Les entreprises familiales doivent établir des canaux de communication pour que tous les membres de la famille soient au courant de l'activité, de la stratégie de la direction générale vers laquelle l'entreprise tend.

\section{Les caractéristiques de la gouvernance dans l'entreprise familiale:}

Les entreprises familiales sont des entités complexes dans lesquelles les rôles respectifs de la famille, du management et de l'actionnariat sont souvent confondus. Elles représentent des organisations particulières nécessitant une gouvernance particulière (Nordqvist et Melin (2002).

La famille peut contribuer à l'entreprise en lui transmettant sa propre culture, c'est-à-dire un ensemble de valeurs fortes décollant de la famille. Le conseil d'administration qui reçoit cette culture, en retour, va faire en sorte que les stratégies, les décisions et les actions qui seront mises en œuvre s'alignent sur les attentes et les valeurs de la famille. Ainsi, un bon système de gouvernance conduit à une plus grande clarté des rapports, à la fois au sein de la famille, et entre la famille et le conseil d'administration et concoure à rendre plus fortes l'entreprise et la famille.

Dans l'ensemble, il s'agit d'un très important travail de collation entre les deux structures de gouvernance (l'entreprise et la famille), notamment lorsqu'il s'agit des questions auxquelles la famille est particulièrement sensible.

Dans une entreprise familiale développée, cela implique une vision claire de la manière dont l'entreprise et sa gouvernance sont structurées, ainsi que des interactions du conseil d'administration et du comité exécutif avec la famille et sa structure. Ceci est illustré dans la figure suivante telle que présentée par Kenyon- Rouvinez et Ward (2004) : 


\section{Figure $\mathrm{N}^{\circ} 1$ : Représentation graphique d'un système de gouvernance d'une entreprise familiale}

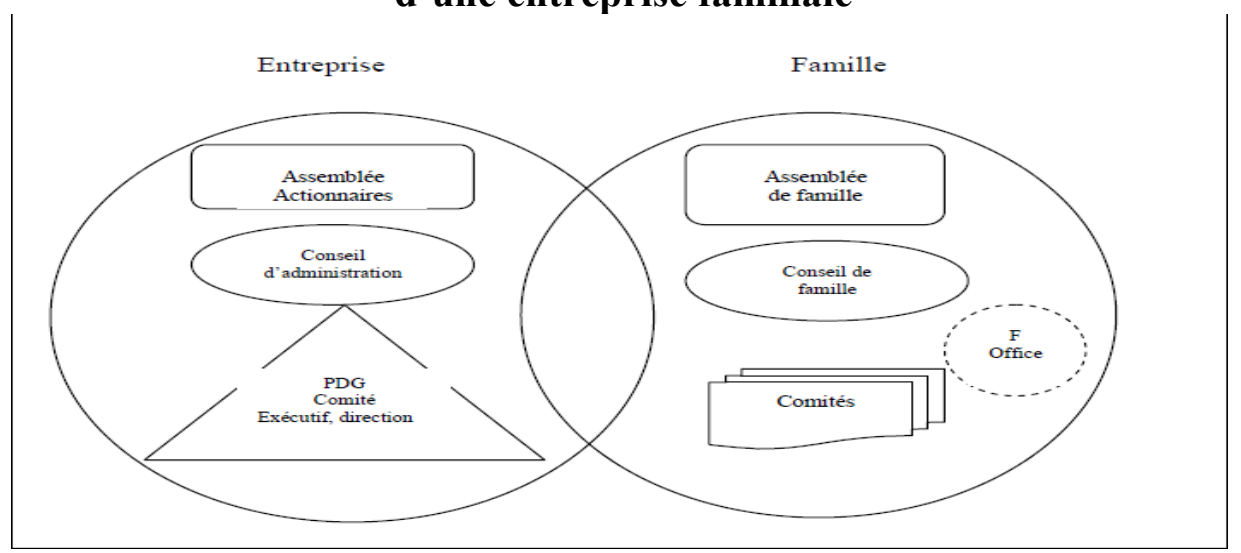

Source : Kenyon- Rouvinez et Ward (2004)

De ce fait, la gouvernance familiale décline en la réalisation, le maintien et le renforcement de l'unité familiale à la fois entre ses membres et avec l'entreprise. La promotion d'un actionnariat stable et motivé et d'une attitude professionnelle des actionnaires à l'égard de l'entreprise afin de ne pas en entraver son fonctionnement.

9. La composition de la structure de la gouvernance familiale:

Dans les entreprises familiales les plus avancées et sophistiquées, le système de gouvernance familiale est organisé autour des structures suivantes :

\section{9-1- La constitution familiale :}

Elle est également désignée comme «charte familiale», qui définit les rôles, les compositions et les pouvoirs des principaux organes de gouvernance de l'entreprise et les relations entre eux. Elle traite principalement les éléments suivants :

- Les valeurs, les missions et la vision familiale ;

- Les institutions familiales;

- Le conseil d'administration et les directeurs ;

- L'autorité, la responsabilité et les relations entre la famille, le conseil d'administration et les directeurs ;

- Les politiques concernant les sujets familiaux importants, tels que l'emploi des membres de la famille, le transfert des actions ...etc.

\section{9-2- Les institutions de la gouvernance familiale :}

-L'assemblée familiale : L'assemblée familiale est une réunion à laquelle sont présents tous les membres «qualifiés » de la famille afin de réfléchir aux domaines d'intérêt commun. La mise en place d'une telle institution leur offre l'opportunité d'exprimer leurs opinions, de renouer leurs relations et de les approfondir et éviter les conflits éventuels qui pourraient surgir entre les membres de la famille à cause d'un accès inégal aux informations et aux autres ressources.

- Le conseil de famille : Appelé également « conseil de surveillance de la famille »il est souvent recommandé. C'est un organe de gouvernance représentatif pour l'assemblée familiale dans la coordination des intérêts des membres de la famille avec leur entreprise. Cet organe est conçu pour remplir ces principales fonctions :

- Etre le lien principal entre la famille, le conseil d'administration et la direction ;

- Suggérer et discuter des noms des candidats pour l'adhésion au conseil d'administration ;

- Elaborer et réviser les documents relatifs à la position de la famille sur sa vision, sa mission et ses valeurs ;

- Elaborer et réviser les politiques familiales, telles que 1'emploi familial, la rémunération et les politiques relatives à la détention d'actions par la famille ; 
- Aborder les autres affaires importantes pour la famille.

- The family office (le bureau familial) : Le bureau familial est un centre d'investissement et d'administration organisé et supervisé par le conseil familial, il mène une activité séparée de l'entreprise, bien que certains de ses membres puissent également travailler dans celle-ci. Le bureau est généralement constitué de gestionnaires qui fournissent des conseils sur la planification d'investissements personnels, la conformité d'assurance, les opérations intrafamiliales et d'autres sujets d'intérêts pour les membres de la famille

- Le comité des actionnaires: Il s'agit d'un organe qui est nécessaire lorsque la famille actionnariale est très étendue. Cet « actionnariat familial dispersé » a besoin de ce comité qui va les représenter dans la structure de la gouvernance. Ce comité a également pour mission de s'occuper de problèmes liés à la vente et le rachat d'actions, les dividendes, les stratégies adoptées par l'entreprise et la mise en œuvre d'une convention d'actionnaires qui a pour objet les questions relatives à l'entrée et à la sortie de l'actionnariat, les droits et devoirs des actionnaires, les systèmes de prise de décisions et de vote.

- Le conseil d'administration dans une entreprise familiale : Dans entreprises familiales, le rôle disciplinaire du conseil est habituellement faible lorsque la famille du dirigeant domine le capital car la discipline est supposée être assurée par l'actionnaire de référence (Ward et Handy, 1988 ; Charreaux, 1996 ; Johannisson et Huse, 2000).

Aldref (1988) a montré que le conseil de l'entreprise familiale doit être externe. Pour lui, les membres de la famille propriétaire sont incapables de percevoir les limites de leurs comportements, la présence des administrateurs externes est donc nécessaire pour limiter les actions pouvant nuire à l'entreprise et due à l'attachement émotionnel des membres de la famille, surtout ceux impliqués dans la gestion.

\section{0- Les faiblesses du système de gouvernance des entreprises familiales:}

Kenyon, Rouvinez et Gallo (2004) ont défini quelques défaillances dans système de gouvernance familiale, à savoir :

- Problèmes liés à certaines spécificités de l'entreprise familiale :

- Le fait que les propriétaires occupent une fonction dans l'entreprise et siègent au même temps au conseil d'administration, ils sont donc pratiquement sur trois fronts à la fois : la propriété, la gestion et la gouvernance.

- La résistance au changement de la part de la famille, donc la mise en place d'un système de gouvernance est pour les membres de la famille « un événement perturbateur ».

- La petite taille de l'entreprise est également un facteur qui limite les possibilités de mettre en place des structures complexes.

- La jeunesse et le manque d'expérience qui caractérise la génération suivante.

- Problèmes liés au conseil d'administration

- Le refus de responsabilité : les membres du conseil d'administration refusent d'assumer la lourde responsabilité qui leur incombe en étant à cette structure de la gouvernance. Ce qui empêche la mise en place de mécanismes de succession, le développement et la revitalisation stratégique de l'entreprise.

- Vouloir sans vouloir, c'est dans le cas où le chef d'une entreprise familiale met en place un conseil d'administration auquel il restreindra le champ de compétence de telle sorte qu'il n'agirait que dans la mesure où il ne serait jamais apte à le contredire. Ceci rendrait alors quasiment inopérant le conseil d'administration, puisqu'il serait écarté des tâches les plus importantes.

- L'instrumentalisation du conseil : dans les entreprises familiales, les réunions du conseil d'administration peuvent être saisies comme des occasions, par certains membres de la famille, pour exercer des pressions et pour orienter les décisions vers des intérêts personnels qui ne concernent pas les objectifs de l'entreprise.

- La fausse équité : les entreprises familiales s'efforcent à composer un conseil d'administration pour lequel la première préoccupation est non pas la performance de l'entreprise mais plutôt la 
Dr.salaouatchi hichem sofiane + Dr ait yahia kamila + E.d. adja hamida

recherche d'une légitimité qui passerait par le respect des droits légaux de tous les actionnaires familiaux.

- Le respect des ainés : le conseil d'administration serait ici composé des ainés de toutes les branches de la famille. C'est en fait, le souci de refléter l'arbre généalogique de la famille qui va manquer ainsi de donner la chance à certains membres pourtant nettement plus compétents.

10. Méthodologie :

Nous optons dans notre recherche à la métohde de l'étude. L épistémologique interprétativiste nous permettra de pouvoir étudier les spécificités de la gouvernance d'entreprise familiale. Nous avons choisi le cas l'entrperise NCA-ROUIBA pour asseoir une méthodologie exploratoire de notre problématique de recherche.

\section{1- Présentation de la NCA-Rouiba :}

NCA-Rouiba est une société privée de droit algérien située sur la Route nationale $\mathrm{N}^{\circ} 5$, Zone industrielle de Rouiba, Alger 16300. Son capital social est de 849195000 DA, elle a été créée en 1966 sous la forme juridique de Société à Responsabilité Limitée (S.A.R.L) sous le nom de «Nouvelles Conserveries Algériennes ». Elle a été transformée en Société Par Actions (S.P.A) au mois de mars 2003. La durée de vie de la société, initialement limitée à 50 ans, a été étendue à 99 ans par l'Assemblée Générale Extraordinaire qui s'est réunie le 5 février 2006.

Depuis sa constitution en 1966, la société n'a exercé que les activités suivantes :

- La production et la distribution de boissons et de jus de fruits ;

- La production et la distribution de conserves de légumes et fruits et du lait UHT (abandonnée en 2005).

Les effectifs de la société sont passés de 336 personnes en 2003 à 479 personnes en 2012 avec une projection de 489 à la fin de l'année 2013, pour atteindre actuellement un effectif de 500 collaborateurs.

\section{2- Organisation de la NCA-Rouiba:}

Afin d'assurer une bonne diffusion des principes de bonne gouvernance d'entreprise au sein de la NCA-Rouiba, la société est dotée d'un organigramme organisationnel clair assorti de fiches de fonction détaillées et adossées à un manuel de procédures et de contrôle interne formel. La NCARouiba est organisée fonctionnellement selon l'organigramme hiérarchique matriciel.

\section{3- Les structures de gouvernance :}

- l'assemblée générale (AG) :

L’Assemblée Générale est composée des actionnaires ou de leurs représentants légaux juridiquement capables. Le capital social de NCA-Rouiba est détenu et réparti entre 19 actionnaires comme le montre le tableau suivant :

Tableau $\mathrm{N}^{\circ}$ 1: La répartition du capital social de la NCA-Rouiba

\begin{tabular}{|l|c|c|}
\hline \multicolumn{1}{|c|}{ Désignation } & $\begin{array}{c}\text { Nombre } \\
\text { d'actions }\end{array}$ & $\%$ \\
\hline Salah OTHMANI & 10 & $0,0 \%$ \\
\hline Salah OTHMANI (usufruitier) et Slim OTHMANI (nu-propriétaire) & 263990 & $3,1 \%$ \\
\hline Slim OTHMANI & 2660330 & $31,3 \%$ \\
\hline Mohamed El Hadi OTHMANI & 654830 & $7,7 \%$ \\
\hline Thouraya OTHMANI & 399450 & $4,7 \%$ \\
\hline Mohamed ATHMANI & 360160 & $4,2 \%$ \\
\hline Mohamed Saïd Aymen ATHMANI & 300000 & $3,5 \%$ \\
\hline Saïd ATHMANI & 154810 & $1,8 \%$ \\
\hline Saïd ATHMANI (usufruitier) et Adnan ATHMANI (nu-propriétaire) & 100000 & $1,2 \%$ \\
\hline Saïd ATHMANI (usufruitier) et Dorra ATHMANI (nu-propriétaire) & 100000 & $1,2 \%$ \\
\hline Mohamed Sahbi OTHMANI & 72030 & $0,8 \%$ \\
\hline Mohamed Mokhtar OTHMANI & 70000 & $0,8 \%$ \\
\hline Khadija OTHMANI & 55660 & $0,7 \%$ \\
\hline Zazia OTHMANI & 55660 & $0,7 \%$ \\
\hline Faouzia ATHMANI & 55660 & $0,7 \%$ \\
\hline
\end{tabular}


Dr.salaouatchi hichem sofiane + Dr ait yahia kamila + E.d. adja hamida

\begin{tabular}{|l|c|c|}
\hline Yamina ATHMANI & 50000 & $0,6 \%$ \\
\hline Lamy Mohamed SEKKARA & 5660 & $0,1 \%$ \\
\hline Mohamed Mehdi OTHMANI & 2030 & $0,0 \%$ \\
\hline Adnane ATHMANI & 10 & $0,0 \%$ \\
\hline Dorra ATHMANI & 10 & $0,0 \%$ \\
\hline Africinvest Fund Ltd & 3131640 & $36,9 \%$ \\
\hline Maghrebinvest Algérie SPA & 10 & $0,0 \%$ \\
\hline TOTAL actions : & 8491950 & $100 \%$ \\
\hline MONTANT CAPITAL SOCIAL: & 849195000 DA \\
\hline
\end{tabular}

Source : document interne de la NCA Rouib

- Résultat conclu à propos de l'AG :

- En termes de participation aux assemblées, l'ensemble des actionnaires a le droit d'assister aux réunions des Assemblées Générales. Aussi, les informations complètes relatives à la tenue des assemblées arrivent en temps opportun et utile aux actionnaires. Les points de l'ordre du jour sont documentés, au préalable et les actionnaires disposent du droit effectif d'inscrire de points à l'ordre du jour. Aussi, l'entreprise prévoit, en sus de l'obligation légale de publication dans un journal qualifié, de mettre en ligne l'avis de convocation sur son site Internet.

- En termes d'accès à l'information, les actionnaires accèdent aux informations relatives aux comptes de l'entreprise, aux rapports des commissaires aux comptes, et aux autres expertises.

- En termes de distribution des dividendes, les actionnaires ont un droit effectif de décision sur la répartition des dividendes (politique explicite de distribution) et ils disposent en temps utile des choix stratégiques et informations pouvant affecter la distribution des dividendes.

- En termes de gestion des conflits d'intérêts, l'ensemble des transactions entre parties liées sont soumises à l'approbation des actionnaires et font l'objet d'un rapport spécial du commissaire aux comptes.

- Le Conseil d'Administration (CA):

Le conseil d'administration de NCA-Rouiba est composé de 11 administrateurs élus par l'assemblée générale ordinaire tenue le 7 juin 2010 pour une durée de 6 ans. Le conseil d'administration a élu parmi ses membres Monsieur Slim OTHMANI en qualité de président lors de sa réunion tenue le même jour.

Le conseil d'administration contrôle la majorité du capital social de la société. Ses membres détiennent plus de $90 \%$ du capital, ce qui couvre le seuil minimum de $20 \%$ prévu par les dispositions du code de commerce.

Tableau $\mathbf{N}^{\circ}$ 2: Composition actuelle du conseil d'administration de la NCA-Rouiba

\begin{tabular}{|c|c|c|}
\hline Nom et prénom & Fonction & Mandat \\
\hline Salah OTHMANI & $\begin{array}{l}\text { Président } \\
\text { d'honneur }\end{array}$ & \multirow{8}{*}{$\begin{array}{l}\text { Mandat de six ans à partir du } \\
29 \text { juin } 2009 \text { et devant } \\
\text { expirer à l'occasion de } \\
\text { l'AGO statuant sur les } \\
\text { comptes de } 2015\end{array}$} \\
\hline Slim OTHMANI & Président & \\
\hline Mohamed Sahbi OTHMANI & $\begin{array}{l}\text { Membre- } \\
\text { directeur } \\
\text { général }\end{array}$ & \\
\hline Saïd ATHMANI & Membre & \\
\hline $\begin{array}{l}\text { Mohamed El Hadi } \\
\text { OTHMANI }\end{array}$ & Membre & \\
\hline Mohamed ATHMANI Membre & Membre & \\
\hline $\begin{array}{l}\text { Africinvest Fund représentée } \\
\text { par Mohamed Aziz } \\
\text { MEBAREK }\end{array}$ & Membre & \\
\hline $\begin{array}{l}\text { Maghrebinvest Algérie SPA } \\
\text { représentée par Mehdi } \\
\text { GHARBI }\end{array}$ & Membre & \\
\hline Thoraya OTHMANI & Membre & $\begin{array}{l}\text { à partir du } 7 \text { juin } 2010 \text { et } \\
\text { devant expirer de } 1 \text { 'AGO }\end{array}$ \\
\hline
\end{tabular}


les specificites de la gouvernance des entreprises familiales cas : nca rouiba

Dr.salaouatchi hichem sofiane + Dr ait yahia kamila + E.d. adja hamida

\begin{tabular}{|l|l|l|}
\hline & & statuant sur les comptes de 2015 \\
\hline Zaher MESSAOUDI & Membre & $\begin{array}{l}\text { Nommé le18 octobre 2012 et prendra ses fonctions dès } \\
\text { l'acquisition d'une action de garantie (au moins) }\end{array}$ \\
\hline $\begin{array}{l}\text { M. Jean Pierre } \\
\text { MONTALIVET }\end{array}$ & $\begin{array}{l}\text { Observateur } \\
\text { indépendant }\end{array}$ & $\begin{array}{l}\text { à partir du 8 août 2011 et devant expirer à l'occasion } \\
\text { de l'AGO statuant sur les comptes de 2015 }\end{array}$ \\
\hline
\end{tabular}

Source : document interne de la NCA-Rouiba

- Résultat conclu à propos du CA :

Afin de pouvoir définir les rôles et les responsabilités des membres du conseil d'administration, la société s'est dotée d'un règlement intérieur du conseil d'administration sur recommandation des équipes Middle East and North Africa, Corporate Governance de 1'IFC (Groupe Banque Mondiale). Le rôle du Conseil vis-à-vis du top management est clairement défini et la séparation de la fonction de directeur général de la fonction de président du conseil d'administration est effective depuis 2003.

En ce qui concerne la composition du conseil d'administration, la société bénéficie d'une représentativité élargie avec trois membres du management de la société, de six membres représentant les actionnaires et de deux administrateurs indépendants, dont un assiste en tant qu'observateur. Le secrétariat du conseil a été confié à un cabinet d'avocats indépendant. Et en ce qui concerne son fonctionnement, le conseil se réunit au moins une fois par an sur convocation officielle avec un ordre du jour préétabli.

En termes de décisions, en essayant de savoir quelles types de décisions concernent le conseil d'administration on a pu constater que le décideur réel pour la réorganisation de l'entreprise, le recrutement d'un membre de l'équipe dirigeante est l'équipe dirigeante et non le conseil, aussi, le domaine de la stratégie de l'entreprise apparait être la chasse gardée de l'équipe dirigeante. En revanche, les opérations structurelles telles que la cession d'actifs ou la rémunération des dirigeants sont plus balancées entre les décisions des dirigeants et du conseil, ainsi, le pouvoir de décision opérationnelle est concentré entre les mains des dirigeants sans qu'un besoin de contrôle ne se fasse ressentir. En revanche, le conseil est supposé intervenir dans les moments décisifs de l'entreprise.

- Les comités émanant du conseil d'administration :

Le fonctionnement de la société obéit aux règles de gouvernance d'entreprise qui préconisent la mise en place de comités ad-hoc émanant du conseil dont chacun est investi d'une mission spécifique.

La société s'est dotée d'un comité de la stratégie et des investissements depuis l'entrée de la société de capital investissement non résidente Africinvest - Fund dans son capital qui se réunit mensuellement et fait l'objet d'un procès-verbal. D'autres comités du conseil sont créés ou encours de création depuis 2010, il s'agit notamment du comité d'audit, du comité des nominations, du comité de rémunération et du comité pour l'éthique, l'environnement et le développement durable.

- La direction de la NCA Rouiba :

Assurée par Mohamed Sahbi OTHMANI qui occupe le poste de Directeur Général de la société depuis 2010.

- Les pactes d'actionnaires : Il existe deux pactes d'actionnaires régissant les relations entre certains actionnaires.

- Résultat à propos du pacte des actionnaires :

*Un pacte d'actionnaires conclu entre Monsieur Salah OTHMANI, Monsieur Saïd ATHMANI, Monsieur Mohamed El Hadi OTHMANI, Monsieur MohamedATHMANI, Monsieur Slim OTHMANI, Madame Thouraya OTHMANI et Africinvest du 21 février 2005, et modifié par un avenant le 28 mars 2005, contient les principales stipulations suivantes :

- L'engagement des actionnaires de la famille OTHMANI, parties de ce pacte, à désigner Africinvest comme administrateur et à maintenir cette désignation durant toute la période de validité du pacte ;

- Un droit d'audit permettant à Africinvest de charger un expert-comptable d'effectuer des missions de contrôle comptable et financier. 
Dr.salaouatchi hichem sofiane + Dr ait yahia kamila + E.d. adja hamida

- Une clause de liquidité prévoyant l'introduction de la société en Bourse ou à défaut le rachat de la participation d'Africinvest par les actionnaires parties de ce pacte sur la base de paramètres de valorisation et d'un calendrier préétablis ;

- Une clause prévoyant un droit de retrait au bénéfice d'Africinvest avec des conditions financières spécifiques en cas :

- De perte de la position de contrôle par les actionnaires parties du pacte;

- Du non-respect de certaines obligations incluant l'obligation de non-concurrence ou de l'intérêt de la société, de respects des règles relatives aux conflits d'intérêt ;

- De non reconduction de la désignation d'Africinvest au Conseil d'administration.

*Un pacte d'actionnaires conclu le 17 avril 2012 pour une durée de trois ans entre Slim OTHMANI, Mohamed El Hadi OTHMANI, Mohamed ATHMANI, Thouraya OTHMANI épouse Kamoun, Mohamed Sahbi OTHMANI, contient les principales stipulations suivantes :

- Les parties de ce pacte détenant ensemble la majorité relative du capital de la Société ont décidé de se constituer en noyau dur d'actionnaires afin d'assurer une stabilité du management de la société dans le but d'atteindre les objectifs tracés dans le business plan de

NCA-Rouiba. Ces parties ont donc convenu d'organiser et de régir leurs relations d'actionnaires à compter de l'introduction en Bourse de la Société.

- Le pacte prévoit l'institution d'un Conseil de Famille investi d'une mission de concertation préalable aux assemblées générales de la Société et qui se réunit autant que de besoin à l'effet de discuter des enjeux liés :

- A la relation entre le Noyau Dur et la Société ;

- Aux rapports entre la famille OTHMANI et la Société ; et plus généralement ; à la cohésion du Noyau Dur constitué en vertu des présentes.

Le pacte prévoit l'incessibilité pendant trois ans des actions détenues par les membres du noyau dur à l'exception des cessions entre les membres du noyau dur qui sont, elles, autorisées sous réserve du respect de la règlementation en vigueur, régissant les transactions de bloc.

\section{Discussion sur la gouvernance familiale de la NCA-Rouiba :}

Dans notre cas, la NCA-Rouiba est une entreprise familiale qui est à sa troisième génération. Et qui est passée par plusieurs étapes avant d'arriver là où elle est aujourd'hui, en effet, elle a pu passer le cap de deux générations en gardant son caractère familial.

La NCA-Rouiba a passé le cap de la première génération, l'étape du fondateur, cette étape s'est caractérisée par un fort engagement de la part des fondateurs Salah Othmani et son père

Mohamed Saïd Othmani qui détenaient chacun $83 \%$ et $17 \%$ des parts envers le succès de l'entreprise et ce par leur qualité d'entreprenariat ainsi que leur volonté de participer au développement du pays en créant de la valeur ajoutée.

Les fondateurs ont géré l'entreprise de 1966-1978. A sa création, c'était une petite entreprise familiale qui fabriquait des conserves de fruits et légumes et il fallait s'adapter au contexte de l'époque, l'Algérie venait de recouvrir son indépendance, les investissements étaient contrôlés par l'Etat, la production de l'entreprise était totalement vendue au réseau de distribution de l'Etat. De ce fait, l'entreprise évoluait mais ne grandissait pas beaucoup.

Durant cette période, les questions de gouvernance n'étaient pas vraiment posées puisque le capital est concentré entre les mains des fondateurs, toutes les décisions étaient prises par les fondateurs. De plus, à l'époque le dispositif législatif, réglementaire et institutionnel régissant les entreprises n'était pas encore vraiment établit.

En 1978, Mohamed Saïd Othmani a cédé ses parts à ses enfants Mokhtar, Saïd, El-Hadi, Hassane et Mohamed, ainsi Salah a été rejoint de ses frères composant ainsi la deuxième génération, l'entreprise est passé à l'étape du partenariat fraternel, les frères Othmani ont géré l'entreprise de 1978 à 1999.

Durant cette période, grâce aux allègements et assouplissements en matière d'investissements, le marché commençait à se développer, l'entreprise a commencé à diversifier ses 
produits, en plus des conserves des fruits et légumes elle s'est mise à produire des jus de fruits, des boissons aux fruits et des nectars aux fruits, aussi, une nouvelle ligne d'emballage, la ligne

Tetrapack pour conditionner les jus a été adopté, NCA-Rouiba est la première société en Algérie à adopter ce type de conditionnement. De plus, au début des années 90, Slim Othmani l'actuel président du conseil d'administration a rejoint l'entreprise et avec son aide NCA-Rouiba est devenue l'embouteilleur de Coca Cola en Algérie sous l'enseigne Fruital.

Cette période a été particulièrement difficile pour l'entreprise, les problèmes sont devenus plus complexes que ceux observés pendant l'étape des fondateurs. En effet, du fait de l'augmentation le nombre d'actionnaires, l'entreprise s'est retrouvée dans le cas d'une gestion multiple sans ligne directrice claire et unique puisque tous les membres de la famille étaient décisionnaires. Cela a perturbé les employés et l'entreprise s'est retrouvée dans une situation d'immobilisme total pour peu que les directives étaient différentes. De plus le patrimoine de l'entreprise n'était pas dissocié du patrimoine de la famille ainsi chaque membre de la famille se donnait le droit de demander ce qui était une situation inacceptable.

De plus, l'entreprise avait une culture patriarcale, les ainés avaient tous les pouvoirs et cela a induit un malaise pour la nouvelle génération puisque c'était le lien familial qui primait sur la compétence et le savoir- faire.

Les résultats de ces erreurs de gestion sont vite arrivés et l'entreprise a dû céder Fruital qui était à l'époque la plus grande entreprise du groupe et cela parce qu'elle n'a pas su gérersereinement le volet familial de l'organisation. Et c'est là qu'est apparue la nécessité de la mise en place des pratiques de gouvernance.

De 1999 jusqu'à aujourd'hui, le relais a été passé à la troisième génération, Slim Othmani est nommé directeur général de la NCA-Rouiba en 1999, depuis il révolutionné l'entreprise, en effet, en tirant des leçons des erreurs de gestion de la génération précédente, Slim Othmani a décidé de gérer l'entreprise de façon différente : Faire primer la compétence sur l'âge ;

Limiter le nombre des membres actifs de la famille ; Faire venir des talents de l'extérieur ;

Devenir plus transparents avec toutes les parties prenantes, actionnaires, banques, fournisseurs, l'Etat, etc...et cela ne pouvait se faire sans la mise en place des pratiques de gouvernance. Même si les membres de la famille étaient réticents à l'idée au début, la croissance soutenue qu'a connue l'entreprise 1999-2004 grâce à la mise en place des pratiques de gestion les a convaincus. La gouvernance d'entreprise a été renforcée d'abord avec l'entrée du fonds d'investissement dans le capital de l'entreprise en 2005. La décision d'entrée en bourse en 2010 n'a fait que renforcer la gouvernance d'entreprise de la NCA-Rouiba.

Lors de notre étude, on a pu constater que le fonds d'investissement jouait un rôle important dans la gouvernance familiale de l'entreprise en servant d'arbitre entre les actionnaires actifs et non actifs.

Le recrutement des membres de la famille était basé sur la compétence et non sur le népotisme, il n'y a pas de privilèges pour les membres de la famille, d'autant plus qu'il n'y a que deux membres de la famille qui travaillent dans l'entreprise, Slim Othmani, président du conseil d'administration et Sahbi Othmani, directeur général, les autres membres sont soit membre du conseil d'administration soit des actionnaires non actifs.

Aussi, les relations entre les actionnaires sont régies par un ensemble de règles non écrites. En 2012, un pacte d'actionnaires a été conclu entre les membres de la famille détenant ensemble la majorité du capital, constituant ainsi un noyau dur et ce afin d'assurer une stabilité du management de l'entreprise. Ce pacte a prévu l'institution d'un conseil de famille investi d'une mission de concertation préalable aux assemblées générales de la société et qui se réunit pour discuter des relations entre le noyau dur et l'entreprise, des rapports entre la famille Othmani et l'entreprise.

Mais, l'entreprise n'a pas encore mit en place des structures de gouvernance familiale de façon formelle réunissant tous les membres de la famille actionnaires, telles que une assemblée familiale ou un conseil de famille nécessaires pour préserver les relations des actionnaires familiaux. En effet, la famille Othmani a atteint une taille considérable et ses membres appartiennent à des générations 
différentes ainsi ils peuvent avoir des idées divergentes. Ainsi, le besoin de structures où peuvent s'exprimer les attentes de la famille devient plus grand.

Dans notre étude, nous avons pu constater aussi que les membres de la famille Othmani, avaient des avis divergents sur notamment le recrutement et la promotion des membres de la famille, la distribution des bénéfices, l'intégration de nouveaux actionnaires non familiaux ainsi que les investissements de l'entreprise. C'est pour cela qu'il nous a paru important pour l'entreprise de mettre en place des structures de gouvernance familiale qui permettront de renforcer l'harmonie et les relations familiales avec l'entreprise et de résoudre les conflits familiaux auxquels l'entreprise fait face et qui pourraient perturber son fonctionnement.

\section{Conclusion}

A travers cette contribution, nous avons pu constater que les entreprises familiales sont considérées comme une des principales sources de croissance économique. En raison de leur nature, ces dernières font face à de nombreux défis supplémentaires par rapport à ceux que leurs équivalents doivent affronter. Certains de ces défis peuvent être résolus en adoptant une structure de gouvernance d'entreprise adéquate au sein de l'entreprise. Ainsi le système de gouvernance de l'entreprise familiale Algérienne présente des caractéristiques bien distinctes, car en plus des bienfaits de la mise en place d'un système de gouvernance reconnu pour toute entreprise, il y a également le souci de l'organisation et l'harmonie de la famille comme enjeu.

L'étude de ce cas particulier nous a permis de constater que la NCA-Rouiba se positionne au niveau des plus grandes entreprises algériennes en termes de gouvernance familiale, cette dernière a résisté au temps, en s'inscrivant toujours dans une logique de performance et d'amélioration continue et en réussissant à faire face à d'importants changements, tels que le changement générationnel, l'ouverture du capital de l'entreprise, la réussite de son introduction en bourse en 2013 tout en gardant son caractère familial

La capacité de NCA-Rouiba à dépasser toutes ces étapes cruciales, a été facilitée par la mise en place des pratiques de gouvernance à l'avant-garde portées par des valeurs familiales historiques et une culture d'entreprise profondément ancrée.

En revanche, l'entreprise n'a pas encore mit en place des structures de gouvernance familiale telles qu'un conseil de famille ou une assemblée familiale de façon formelle. De cette façon, la gouvernance familiale, qui traite de la structure et du devenir de son patrimoine n'est pas encore dissociée de la gouvernance d'entreprise, cette confusion des genres est sans doute une caractéristique sur laquelle il faudra porter l'attention.

\section{Bibliographie}

- ELMANNA MADANI (W), l'efficacité du conseil d'administration dans les entreprises familiales, thèse de doctorat en sciences comptables, université de la Manouba, 2010.

- GHUEDDACHE (L), étude de comportement stratégique de l'entreprise familiale, thèse de doctorat en sciences économiques, université mouloud Mammeri de Tizi-Ouzou, 2012.

- KHERRAF $(\mathrm{H})$, la gouvernance des entreprises familiales, mémoire de magister en management, option : finance, université d'Oran, 2014.

- NEUBAUER (F) et LANK (A.G), " the family business: its governance for sustainability", Routledge New York, 1998.

- Société financière internationale (Groupe de la Banque Mondiale). «Manuel de gouvernance des entreprises familiales » Washington, DC, USA, 2008.

- BARRÉDY (C) ET BATAC (J) "Le rôle des dynamiques familiales dans la stratégie et la gouvernance des entreprises : soixante ans d'histoire d'une entreprise familiale dans Annales des Mines, 2013/1 ( $\left.{ }^{\circ} 111\right)$.

- ROUVINEZ (D.K) ET WARD (J), "Les entreprises familiales", édition presses universitaires de France, 2004.

- http://www.bea.dz/pdf/notice_NCA\%5B1\%5D.pdf

- http://www.strategie-aims.com/events/conferences/3-xviiieme-conference-de-laims/communications/236conseil-dadministration-dans-les-entreprises-familiales-eclairageempirique/download. 